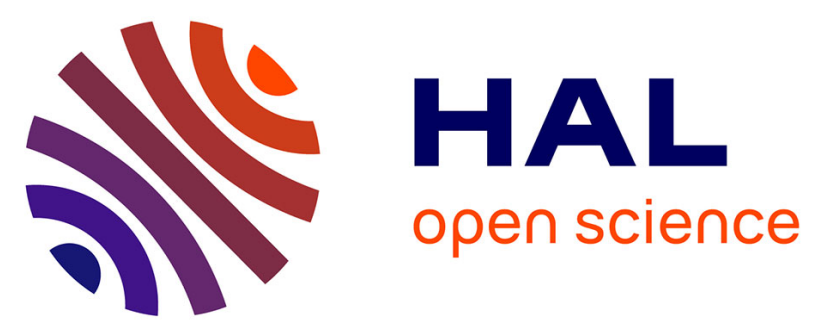

\title{
Disentangling Heat and Moisture Effects on Biopolymer Mechanics
}

\author{
Chi Zhang, Ali Shomali, Robert Guyer, Sinan Keten, Benoit Coasne, \\ Dominique Derome, Jan Carmeliet
}

\section{- To cite this version:}

Chi Zhang, Ali Shomali, Robert Guyer, Sinan Keten, Benoit Coasne, et al.. Disentangling Heat and Moisture Effects on Biopolymer Mechanics. Macromolecules, 2020, 53 (5), pp.1527-1535. 10.1021/acs.macromol.9b01988 . hal-02990008

\section{HAL Id: hal-02990008 https://hal.science/hal-02990008}

Submitted on 5 Nov 2020

HAL is a multi-disciplinary open access archive for the deposit and dissemination of scientific research documents, whether they are published or not. The documents may come from teaching and research institutions in France or abroad, or from public or private research centers.
L'archive ouverte pluridisciplinaire HAL, est destinée au dépôt et à la diffusion de documents scientifiques de niveau recherche, publiés ou non, émanant des établissements d'enseignement et de recherche français ou étrangers, des laboratoires publics ou privés. 


\section{Disentangling Heat and Moisture Effects on}

\section{Biopolymer Mechanics}

3 Chi Zhang $^{\sharp * *}$, Ali Shomali ${ }^{\dagger}$, Robert Guyer ${ }^{*}$, Sinan Keten ${ }^{\S}$, Benoit Coasne", Dominique Derome,

4 Jan Carmeliet"

5 Chair of Building Physics, Department of Mechanical and Process Engineering, ETH Zurich,

6 8093, Zurich, Switzerland

$7 \quad{ }^{\ddagger}$ Laboratory for Multiscale Studies in Building Physics, Swiss Federal Laboratories for Materials

8 Science and Technology, Ueberlandstrasse 129, 8600, Duebendorf, Switzerland

$9 *$ Department of Physics, University of Nevada, Reno, 1664 N. Virginia Street, Reno, NV, 89557,

10 United States

$11{ }^{\S}$ Department of Civil and Environmental Engineering, Northwestern University, 2145 Sheridan

12 Road, Evanston, Illinois, 60208-3109, United States

13 'Univ. Grenoble Alpes, CNRS, LIPhy, 38000 Grenoble, France

14

15 Abstract 
16 Heat and moisture are known to have important mechanical effects on polymers such as hygric

17 swelling, thermal expansion and mechanical weakening. A common approach when

18 investigating such effects is to assume the effects of heat and moisture to be similar - the so-

19 called time-temperature-moisture superposition. Through molecular dynamics simulation, this

20 study evaluates the extent of the similarity of the effects of moisture and heat on the hygric

21 swelling, thermal expansion and mechanical weakening of a biopolymer: an uncondensed type of

22 lignin, one of the most abundant polymers in the plant regime. We introduce as a microscopic

23 metric the local stiffness $\left(T /\left\langle u^{2}\right\rangle\right.$, temperature divided by the amplitude of segmental motion

$\left.24\left\langle u^{2}\right\rangle\right)$, to analyze the mechanisms of mechanical effects of heat and moisture. The local stiffness

25 of polymer skeleton and the overall stiffness of the composite material are shown to be strongly

26 correlated, with a Pearson correlation coefficient of 0.96 . Under the assumptions of harmonic

27 vibration and isotropy, an explicit equation relating bulk moduli and the local stiffness can be

28 derived, and the theoretically predicted moduli are in good agreement with measurement. The

29 thermal expansion and weakening are shown to be related to each other and both dependent on

30 the local stiffness. The analysis of the potential energy further points out that heating weakens

31 both primary and secondary bonds of the polymer skeleton, while hydration only affects the

32 secondary bonds. This major difference is thought to be the origin of the different impacts of

33 heat and moisture on biopolymer mechanics, offering a different view of the time-temperature-

34 moisture superposition principle. 
37 The influence of heat and moisture on the mechanics of polymers is a fundamental issue with 38 particular relevance to many industrial applications such as textiles, packaging, food science, and

39 building materials. The systematic understanding of the coupled physics of moisture and heat is 40 important for effective utilization of many natural and synthetic polymeric materials.

41 For a large variety of polymers establish the time-temperature superposition principle (TTSP),

42 also named the thermo-rheologically simple postulate, meaning that an increment in temperature

43 is equivalent to an increment of time of observation. The shapes of the elastic moduli vs. time

44 curves under different temperature conditions assemble each other, except for a horizontal shift.

45 In fact, in addition to temperature, a number of other shifting factors are known, such as moisture

46 content, diluent concentration, blend ratios and etc. Varying jointly moisture content and

47 temperature creates a "doubly shifted" master curve, based on which a number of experimental

48 reports proposed the so-called time-temperature-moisture superposition principle (TTMSP) of

49 polymers. ${ }^{1-7}$ This master curve also suggests that the influence of heat and moisture can be

50 treated using mathematical forms similar to the Williams-Landel-Ferry (WLF) equation, ${ }^{1,8,9}$

51 though it is reported that the TTMSP may not hold for low moisture content. ${ }^{10}$ The moisture

52 content is sometimes referred to as "apparent temperature", where an increment in moisture

53 content is taken as an equivalent of the rise of temperature. ${ }^{11}$ The current demonstration of the

54 validity of TTMSP is based on macroscopic and phenomenological observations but lacks

55 observations of the mechanisms at play at microscales. The similar effects of heat and moisture

56 on polymers elastic behavior have yet to be analyzed and their mechanisms at origin elucidated.

57 In this paper, we discuss the heat and moisture effects in terms of their similarities and also

58 their differences, the latter a topic rarely touched in literature. Molecular dynamics (MD), a

59 simulation method widely used in bio-physics or materials science, is chosen as the main tool of 
60 investigation. Applying proper force field parameters and potential equations, MD allows

61 capturing the trajectories of atoms at femtosecond time resolution giving microscopic

62 information. Based on such microscopic movements of atoms, macroscopic variables such as

63 temperature, pressure, enthalpy can be harvested using statistical thermodynamics, making MD a

64 powerful tool for investigating physical and mechanical aspects. Here we focus on the

65 instantaneous elastic response of a model polymeric material.

66 This study uses the uncondensed type of lignin (uLGN) as a prototypical polymer, lignin being

67 one of the most abundant biopolymers on Earth. We recall that uncondensed lignin is a linear

68 polymer, not to be confused with condensed lignin also present in plants, which is randomly

69 cross-polymerized. uLGN is known to be moderately hydrophilic thus serving as a complement

70 to frequently studied hydrophilic polymeric materials, such as nylon (polyamide), ${ }^{12}$ polyvinyl

71 acetate (PVAc) ${ }^{1}$ and polyvinyl alcohol (PVA) ${ }^{4}$. There have been several MD studies focused on

72 various properties of lignin. Petridis et al. study the temperature-dependent structure and

73 dynamics of randomly branched lignin, i.e. condensed type of lignin, and observed that

74 temperature rise enhances dynamics and transits the polymer from compact to extended

75 conformations. ${ }^{13,14}$ Vural et al. combine MD and experiments, namely neutron scattering and

76 dielectric spectroscopy, and found that a hysteresis exists where lignin shows faster dynamics

77 and a larger size at a given temperature when being cooled than being heated. ${ }^{15}$ Buehler et al.

78 investigated the influence of lignin structure on the deformation of wood cell walls. ${ }^{16}$ Beste et al.

79 used the ReaxFF force field to investigate the thermal decomposition in oxygen environment of

80 the most common linkages in softwood. ${ }^{17}$ Despite these works, there is a lack of comprehensive

81 study of the mechanics of lignin under various temperatures and moisture contents. In particular, 
82 a better understanding of the microscopic mechanisms of the combined impact of heat and

83 moisture could help to improve the delignification process, a common pretreatment of biomass.

84 There are few papers discussing the combined influence of heat and moisture on the

85 mechanical behavior of polymers using MD. Xin and Han show that both heat and moisture

86 water reduces the mechanical properties of the cross-linked epoxy. ${ }^{18}$ Vural et al. find a similar

87 effect of either heat or moisture on polymer segmental relaxation. ${ }^{19}$ Suarez-Martinez et al. show

88 that both temperature and water affect the dynamics of the polyelectrolyte chain in a similar way

89 supporting the time--water-temperature superposition principles. ${ }^{7}$ However, the occurrence of a

90 difference in the moisture and heat influence on the mechanical properties of a polymer, be it

91 lignin, as yet to be investigated with MD.

92 Our study aims at understanding the microscopic mechanisms of hygric swelling, thermal

93 expansion and mechanical weakening under hygric and thermal loadings. To perform this study

94 we determine the local stiffness of the polymer skeleton and the adsorbed water at the

95 microscale. Strikingly simple equations relating the amplitude of segmental motion, sometimes

96 referred to as the Debye-Waller factor, and the macroscopic elastic moduli are derived for the

97 first time. We explain the similarities and differences between the effects of heat and moisture on

98 fundamental aspects of a polymer, namely lignin, taking into account the molecular mobility and

99 energetics. The proposed method offers a way to relate microscopic quantities to macroscopic

100 observables. The results, and the proposed methodology, might be important to various

101 applications such as biofuel production, paper making and hygro-thermo-treatment of wood.

102

1032 Modeling methods

1042.1 Preparation of the hydrated polymeric system 
106 units, as shown in Figure 1. The C2, C5 and C6 positions of the monomer are free of substitution

107 while the $\mathrm{C} 3$ position is substituted with a methoxyl group. ${ }^{20}$ We note that the exact structure of

108 lignins in plants is still ongoing investigation due to its complex nature. The polymer uLGN is

109 understood to be of rather linear type, but we note a lack of experimental characterization of pure

110 uLGN material, caused by the difficulties of its extraction without chemical alteration. This

111 computational study of uLGN serves as a helpful complement. The molecular model here

112 contains five chains with a degree of polymerization 100. The degree of polymerization

113 corresponds to experimental reports. ${ }^{21}$

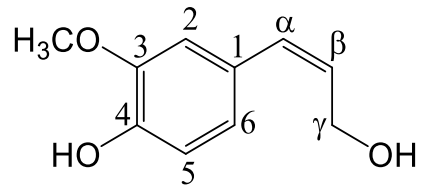

115 Figure 1. Chemical structure of the monomer of uncondensed lignin, coniferyl unit.

116 The initial chemical structure is built in Material Studio 8.0. For MD simulations, Gromacs $1175.0^{22}$ software and Gromos $53 \mathrm{a} 6^{23}$ force field are applied. The Gromos $53 \mathrm{a} 6$ force field is chosen 118 because of its unique feature that it is based primarily on reproducing the free enthalpies of 119 hydration and apolar solvation for a range of compounds, which fits the investigations about 120 biomolecule hydration, solvation and association. ${ }^{23}$ The automated topology builder (ATB) 121 server was used to carry out the parameterization and the primary geometry optimization. ${ }^{24-26}$ 122 The single chains of uLGN are energy minimized. Then, five relaxed chains are placed in one 123 simulation box with full periodic boundary conditions. The system is further relaxed for $20 \mathrm{~ns}$ in 124 the isothermal-isobaric ensemble (NPT) using a Nose-Hoover thermostat and a Parrinello125 Rahman barostat with temperature and pressure being $300 \mathrm{~K}$ and $0 \mathrm{~Pa}$, respectively. The 126 equilibrated system attains a density of $1.33 \mathrm{~g} / \mathrm{cm}^{3}$, which is in accordance with the experimental 
127 result of $1.36 \mathrm{~g} / \mathrm{cm}^{3} .{ }^{27}$ The Herman's orientation function of the equilibrated structure has a value 128 of $0 \pm 0.02$ verifying that the simulated material is isotropic. The hydroxyl groups of lignin are 129 evenly distributed in space, meaning that no hydrophobic or hydrophilic pores are formed. All 130 production simulations are carried out under NPT ensemble. In most simulations, the pressure is 131 set to 0 bar, except for the bulk modulus measurements where the system is tested under a 132 specific mechanical loading. The cut-off distance of both Coulombic and van der Waals 133 interactions is set to $1 \mathrm{~nm}$ and the long-range Coulombic interactions are calculated by particle 134 mesh Ewald summation. The covalent bonds with hydrogen atoms are constrained with the 135 LINCS algorithm. The time step of integration is $1 \mathrm{fs}$.

136 Water molecules are introduced in the system through the random insertion of the single point 137 charge (SPC) model of water, followed by energy minimization and $10 \mathrm{ps}$ of equilibration. The 138 Gromos force field is designed to work with SPC water model. This study focuses on the 139 thermodynamic equilibrium state without the consideration of water diffusion process. The SPC 140 model predicts well the density, heat of vaporization and isothermal compressibility of pure 141 water. However, we note that this model yields a relatively larger value for the thermal 142 expansion coefficient (TEC) than what is measured in experiments. ${ }^{28}$ To reach the equilibrated 143 state with less computational cost, the water molecules are initially inserted into random 144 locations and the following equilibration steps allow attaining thermodynamically favored 145 locations. For each moisture level, an extra $20 \mathrm{~ns}$ of equilibration is applied. The distribution of 146 water molecules, quantified by the radial distribution function of the oxygen atoms of water, is 147 seen to remain stable after $10 \mathrm{~ns}$, meaning that $20 \mathrm{~ns}$ of equilibration time is more than sufficient 148 to reach equilibrium statistically. The polymer carbon atoms radial distribution functions are also 
checked in a similar way. In fact, extended simulations as long as $1000 \mathrm{~ns}$ are carried out and

150 give similar radial distribution function results as the $20 \mathrm{~ns}$ results.

151 The studied moisture content, reported in mass of water per mass of dry polymer, and the 152 temperature range between $0 \sim 30 \%$, with an increment of $4 \%$, and $283 \sim 373 \mathrm{~K}$, with an increment 153 of $10 \mathrm{~K}$ respectively. We note that these ranges are broader than the ranges of common ambient 154 conditions of wood usage in order to allow a more in-depth investigation. We note that, in the 155 temperature range of investigation, no major change of chemical or physical properties, such as 156 pyrolysis of polymer and phase change of water, occurs. The pyrolysis temperature of lignin $157(\sim 600 \mathrm{~K})^{29}$ is much higher than the maximum temperature studied here. Water molecules 158 confined in porous media have a lower freezing point $(<273 \mathrm{~K})^{30}$ and higher boiling point $(>373$ $159 \mathrm{~K})^{31}$ than bulk water due to the Gibbs-Thomson effect. This ensures that, in this work, water 160 molecules do not experience phase changes, i.e. freezing or evaporation.

161 Three repetition systems are prepared following the same modeling process above. They differ 162 by their polymer orientations and initial water insertion locations. All the repetition systems are 163 then tested under the same protocol described below, and the obtained results are averaged with 164 standard deviations indicated.

1652.2 Evaluation of the free strain due to changes in moisture content and temperature

166 The volume of the system, denoted as $V(m, T)$, is measured as the time average volume over a $1670.5 \mathrm{~ns}$ production run that followed a $1.5 \mathrm{~ns}$ equilibration run in the NPT ensemble $(P=0 \mathrm{~Pa})$. 168 The volume is measured under a series of different moisture content and temperature conditions 169 allowing deforming freely. The system reaches its stable state within the first $0.1 \mathrm{~ns}$ of the 170 equilibration run. The volumetric strain $\epsilon_{V}(m, T)$ due to changes in moisture content and 171 temperature is defined as relative difference with respect to the volume at $m=0 \%$ and $T=283 \mathrm{~K}$ : 


$$
\epsilon_{V}(m, T)=\frac{V(m, T)-V(0 \%, 283 \mathrm{~K})}{V(0 \%, 283 \mathrm{~K})}
$$

172 We refer to this volumetric strain as swelling strain when the moisture content is increased, or 173 as thermal expansion strain when the temperature is increased. In general, we will refer to the 174 volumetric strain as free strain.

$175 \quad 2.3$ Evaluation of the bulk modulus

176 The system is equilibrated in NPT $(P=0$ bar) for $20 \mathrm{~ns}$ to obtain the equilibrium volume $177 V(m, T)$ in the free stress state. Then equal pressure on $\mathrm{x}, \mathrm{y}$ and $\mathrm{z}$ directions is applied, where the 178 system is simulated in NPT ( $P=\sigma=500$ bar) for $2 \mathrm{~ns}$ to obtain the equilibrium volume in the 179 loaded state $V^{\sigma}(m, T)$. The system equilibrium is reached within the first $0.1 \mathrm{~ns}$ of the run, 180 which is much shorter than the total relaxation time given to the system of $\sim 2$ ns. The bulk 181 modulus is expressed as: ${ }^{32}$

$$
K(m, T)=-V \frac{\partial \sigma}{\partial V}=\sigma\left|\frac{V(m, T)}{V^{\sigma}(m, T)-V(m, T)}\right|
$$

182 The load value $\sigma$ is chosen to be 500 bar, which is large enough to suppress sufficiently the 183 fluctuation of system volume, and the resulting strain ranges from $2 \%$ to $4 \%$.

$184 \quad 2.4$ Density of the polymer-polymer hydrogen bonds

185 The criteria for hydrogen bond are defined by the configuration of the donor-hydrogen186 acceptor triplet:

$$
r \leq 0.35 \mathrm{~nm} \text { and } \alpha \leq 30^{\circ}
$$

187 where $r$ is the distance between the donor oxygen atom and the acceptor oxygen atom, and $\alpha$ 188 is the angle of acceptor oxygen atom - donor oxygen atom - donor hydrogen atom. The 189 interoxygen distance criterion of $0.35 \mathrm{~nm}$ refers to the first minimum of the radial distribution 190 function of SPC water. ${ }^{33,34}$ The angle of $30^{\circ}$ is approximately the amplitude of vibrations that 
191 break hydrogen bonds. ${ }^{35}$ In this paper, we only discuss polymer-polymer hydrogen bonds. The 192 number of hydrogen bonds is normalized by the volume at $m=0 \%$ and $T=283 \mathrm{~K}$, to eliminate

193 its size dependency, resulting in a Lagrangian density of polymer-polymer hydrogen bonds with 194 a unit of $\mathrm{nm}^{-3}$.

1952.5 Evaluation of the local stiffness $T /\left\langle u^{2}\right\rangle$

196 In this paper, we use the local stiffness as a metric at the microscopic scale. The local stiffness 197 is defined as the quotient of temperature $T$ and the $\left\langle u^{2}\right\rangle$ factor. The $\left\langle u^{2}\right\rangle$ factor indicates the 198 average displacement of atoms at picosecond time scales and is generally assumed to be related 199 to the mechanical properties, i.e. an inverse proportionality is frequently assumed between the $200\left\langle u^{2}\right\rangle$ factor and the local elastic properties, e.g. high-frequency modulus $G_{\infty},{ }^{36}$ derived on the 201 basis of simple Maxwell model of liquids. ${ }^{37}$ The $\left\langle u^{2}\right\rangle$ is also referred to as the Debye-Waller 202 factor, ${ }^{38,39}$ a property of material that can be measured by neutron scattering experiments ${ }^{40}$ and 203 molecular simulations. ${ }^{41}$ On the molecular level, a chain segment displaces itself within a 204 temporary cage formed by its surrounding segments. The MSD of the chain segment keeps 205 increasing until the movement of the segment is restrained by the boundary of the cage and 206 bounces back. A plateau in the MSD-time plot emerges when such rebound happens, where the 207 corresponding time scale is called the caging time and the corresponding $\operatorname{MSD},\left\langle u^{2}\right\rangle$, indicates 208 the size of the molecular cage. Beyond the caging time, the chain segments will show diffusive 209 behavior and escape from the cage.

210 If we assume that the chain segment is confined to its equilibrium location by a harmonic 211 spring, the energy of the chain segment movement can be expressed as $k\left\langle u^{2}\right\rangle$, where $k$ stands for 212 an assumed local spring constant. In this paper, each monomer is taken as a polymer segment 213 and the MSD of its center of mass is measured. Comparing with the MSD of single atoms, the 
214 MSD of the center of mass of the monomer eliminates the contribution of rotation of this

215 monomer to MSD. Within the moisture content and temperature ranges of investigation, the

216 MSD of uLGN polymer plateaus at $\sim 10 \mathrm{ps}$, due to the confinement of the cage formed by the

217 surrounding atoms of the chain segment. Similar to Xia et al. ${ }^{41}$ the $\left\langle u^{2}\right\rangle$ factor in this study is

218 defined as the average mean square displacement (MSD) of polymer segments at $10 \mathrm{ps}$, the

219 caging time of uLGN. The $\left\langle u^{2}\right\rangle$ factor for water is measured similarly, by assessing the average

220 MSD of the center of mass of water molecules.

\section{$221 \quad 3$ Results and discussion}

222 3.1 Macroscopic results: heat and moisture-induced free strain and mechanical weakening

223 Figure 2a shows the free strain as a function of both temperature and moisture content, using a

224 green to yellow scale in addition to contour lines. As moisture content and temperature increases,

225 the free strain increases from 0 at $T=283 \mathrm{~K}$ and $m=0 \%$, to more than 0.4 at $T=373 \mathrm{~K}$ and $m=30$ $226 \%$. 

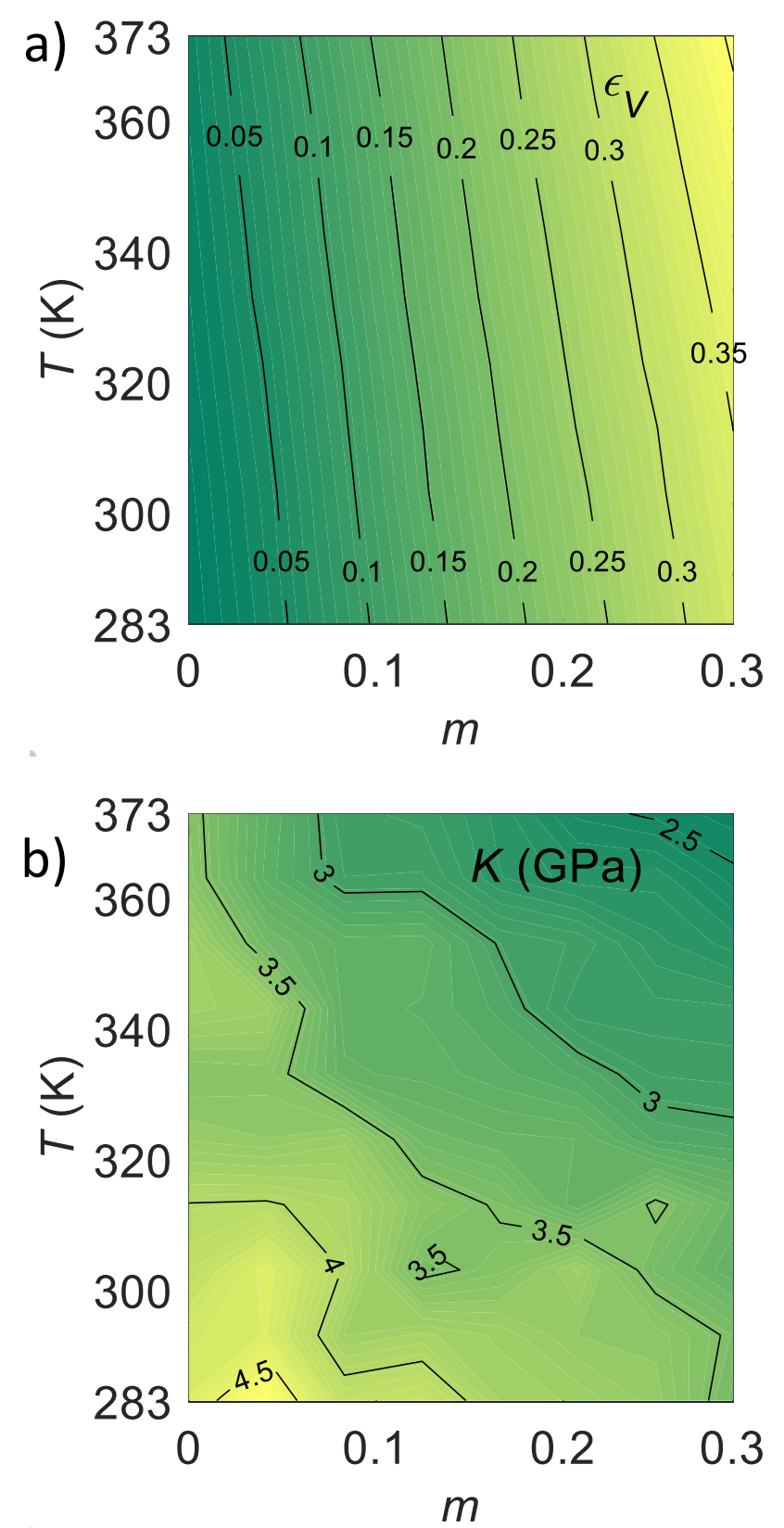

228 Figure 2. a) Free strain as a function of temperature and moisture content. b) Bulk modulus as a 229 function of temperature and moisture content, contour line units are in GPa.

230 The bulk modulus of $\mathrm{uLGN}$ as a function of temperature and moisture content is shown in

231 Figure 2b. The higher moduli are denoted by yellow and the lower by green color. As 232 temperature and moisture content increases, the bulk modulus decreases from more than $4.5 \mathrm{GPa}$ 
233 to less than $2.5 \mathrm{GPa}$ indicating a mechanical weakening of the material. Due to scattering, the 234 contour lines are not as smooth as those for the free strain shown in Figure 2a. The rather 235 diagonal lines indicate that the increase of temperature and moisture content both induce 236 weakening.

237 3.2 Microscopic results: weakening and straining explained by local stiffness $T /\left\langle u^{2}\right\rangle$

238 Figure 3 gives the local stiffness $T /\left\langle u^{2}\right\rangle$ of polymer skeleton and water as a function of 239 temperature and moisture content, respectively. 

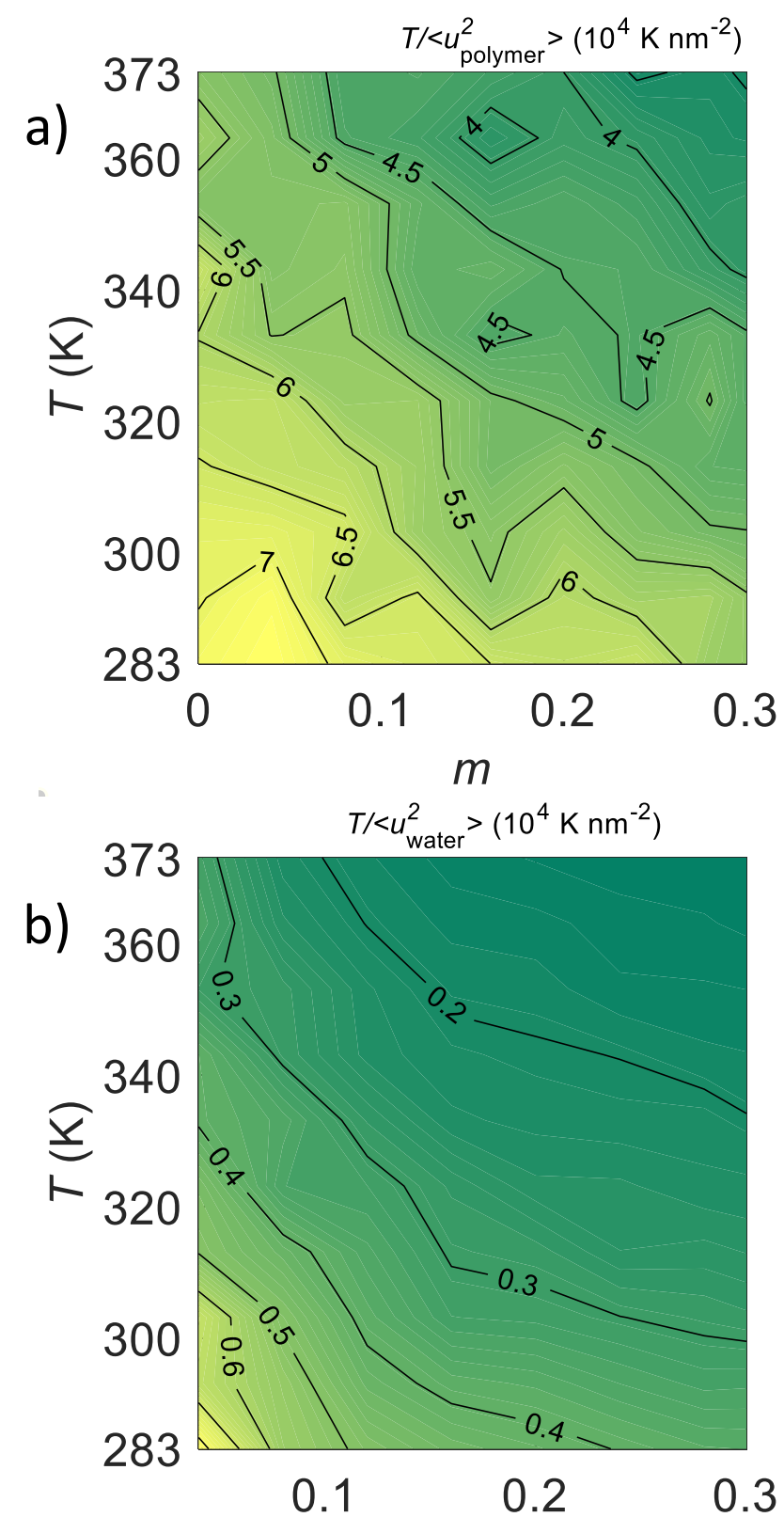

m

241 Figure 3. a) Local stiffness $T /\left\langle u^{2}\right\rangle$ of the polymer skeleton as a function of moisture content $m$

242 and temperature $T$. b) Local stiffness $T /\left\langle u^{2}\right\rangle$ of water as a function of moisture content $m$ and 243 temperature $T$. The unit of the contour lines is $10^{4} \mathrm{~K} \mathrm{~nm}^{-2}$.

244 As shown in Figure 3, $T /\left\langle u^{2}\right\rangle$ for the polymer skeleton and water decrease with increasing 245 temperature and moisture content. In terms of absolute value, the local stiffness of polymer, 
246 ranging from $3.0 \sim 7.410^{4} \mathrm{~K} \mathrm{~nm}^{-2}$, is around 10 times higher than that of water, ranging from

$2470.13 \sim 0.8310^{4} \mathrm{~K} \mathrm{~nm}^{-2}$. This indicates that the stiffness of the hydrated uLGN system is

248 dominated by the stiffness of the polymer chains rather than by the stiffness of the adsorbed

249 water. In fact, the local stiffness of the polymer skeleton (Figure 3a) resembles the overall

250 stiffness of the composite material (Figure 2b), which will be further supported by the correlation

251 analysis in the following sections.

2523.3 Link between microscopic and macroscopic results

253 In this section, we derive a relationship between the local stiffness $T /\left\langle u^{2}\right\rangle$ at the microscale 254 and the macroscopic bulk modulus $K$. The bulk modulus $K$ refers to the isothermal bulk modulus $255 K_{T}$ unless otherwise stated. The time scale studied in this section is in the order of several pico256 seconds, therefore the material is assumed to behave elastically (no time effects). The thermal 257 energy, i.e. the translational kinetic energy, of a chain segment equals $\frac{3}{2} k_{B} T$. If we assume that 258 this chain segment is confined to its equilibrium location by a harmonic spring, the energy of the 259 chain segment movement can be expressed as $k\left\langle u^{2}\right\rangle$, where $k$ stands for an assumed local spring 260 constant. The maximum potential energy stored in the spring should equal the kinetic energy of 261 the segment:

$$
\frac{1}{2} k\left\langle u^{2}\right\rangle \cong \frac{3}{2} k_{B} T
$$

262 The intrinsic vibration frequency of a harmonic spring is $\omega_{0}=\sqrt{k / a \lambda}$, where $a$ is the size of 263 the segment and $\lambda$ the line density of the polymer chain. Therefore, the wave speed in the 264 material is $c=\omega_{0} a$ and we have:

$$
c=\omega_{0} a=\sqrt{k a / \lambda}
$$


The longitudinal wave speed in the material is related to the adiabatic bulk modulus $K_{s}$ (here

266 we assume $K_{S} \cong K_{T}$, which holds for a number of solid materials ${ }^{42}$ ), the shear modulus $G$ and the

267 density of the material $\rho=\lambda a / a^{3}$ :

$$
c=\sqrt{\frac{K_{s}+\frac{4}{3} G}{\rho}}
$$

268 For an isotropic material, the bulk and shear moduli are related by Poisson's ratio through:

$$
G=\frac{3}{2} \frac{K(1-2 v)}{1+v}
$$

269 Combining all these equations we find that the bulk modulus of the dry polymer can be 270 predicted from the local stiffness $T /\left\langle u^{2}\right\rangle$ by:

$$
K=\frac{1+v}{1-v} \frac{k_{B} T}{a\left\langle u^{2}\right\rangle}
$$

271 This equation shows that the bulk modulus at the macroscale is directly related to the 272 amplitude of segmental motion $\left\langle u^{2}\right\rangle$ or local stiffness $T /\left\langle u^{2}\right\rangle$ at microscale.

273 Further, the Young's and shear moduli can be derived for an isotropic material from bulk 274 modulus and Poisson's ratio:

$$
E=\frac{3(1+v)(1-2 v)}{1-v} \frac{k_{B} T}{a\left\langle u^{2}\right\rangle} \quad \text { and } \quad G=\frac{3}{2} \frac{1-2 v}{1-v} \frac{k_{B} T}{a\left\langle u^{2}\right\rangle}
$$

275 The eq. (8 and eq. (9) are strikingly simple, considering that only two assumptions, i.e. 276 harmonic vibration and isotropy, are introduced. The relation between the amplitude of 277 segmental motion $\left\langle u^{2}\right\rangle$ and shear or Young's moduli was shown in, ${ }^{43}$ but in this study explicit 278 equations are provided for bulk, Young's and shear moduli as functions of $\left\langle u^{2}\right\rangle$.

279 Using eq. (8), the bulk modulus of the dry polymer as a function of temperature can be 280 predicted knowing the local stiffness $T /\left\langle u^{2}\right\rangle$ at microscale. Here the chain segment is defined as 281 a lignin monomer. As 500 monomers occupy a volume of around $104 \mathrm{~nm}^{3}$, and given that $500 *$ 
$282 a^{3}=104 \mathrm{~nm}^{3}$, therefore the size of the chain segment is on average $a \sim 0.59 \mathrm{~nm}$. We remark

283 that there is no strict definition of chain segments and other definitions may give different values

284 of chain segment size. For most of the polymers, the Poisson ratio varies between $0.35<v<$

285 0.45. A standalone MD test shows that, at $300 \mathrm{~K}$ and $m=0 \%$, the Poisson's ratio of pure uLGN is

$286 \sim 0.37$, in accordance with experimental estimation. ${ }^{44}$ Figure $4 \mathrm{a}$ is the correlation plot of the

287 measured bulk moduli and the local stiffness of the polymer skeleton. The circles denote the

288 average values of the three repetition tests, and the error bars denote the standard deviation. The

289 color of the circles gradually changes from black to red denoting the increase in temperature. The

290 color of the edges of the circles changes from black to blue denoting the increase in moisture

291 content. The Pearson correlation coefficient of 0.96 indicates that $T /\left\langle u^{2}\right\rangle$ can serve as an

292 indicator for the macroscopic stiffness of the material. We note again that the $\left\langle u^{2}\right\rangle$ can be

293 measured by neutron scattering experiments, ${ }^{40}$ meaning that eq. ( 8 and eq. (9) might be

294 examined by future experimental studies. Though it is known that MD does not reproduce a

295 heating rate equivalent to the experimental one, the correlation between local stiffness and

296 macroscopic stiffness of material is sound because the $\left\langle u^{2}\right\rangle$ and bulk moduli are measured using

297 the same heating protocol. 

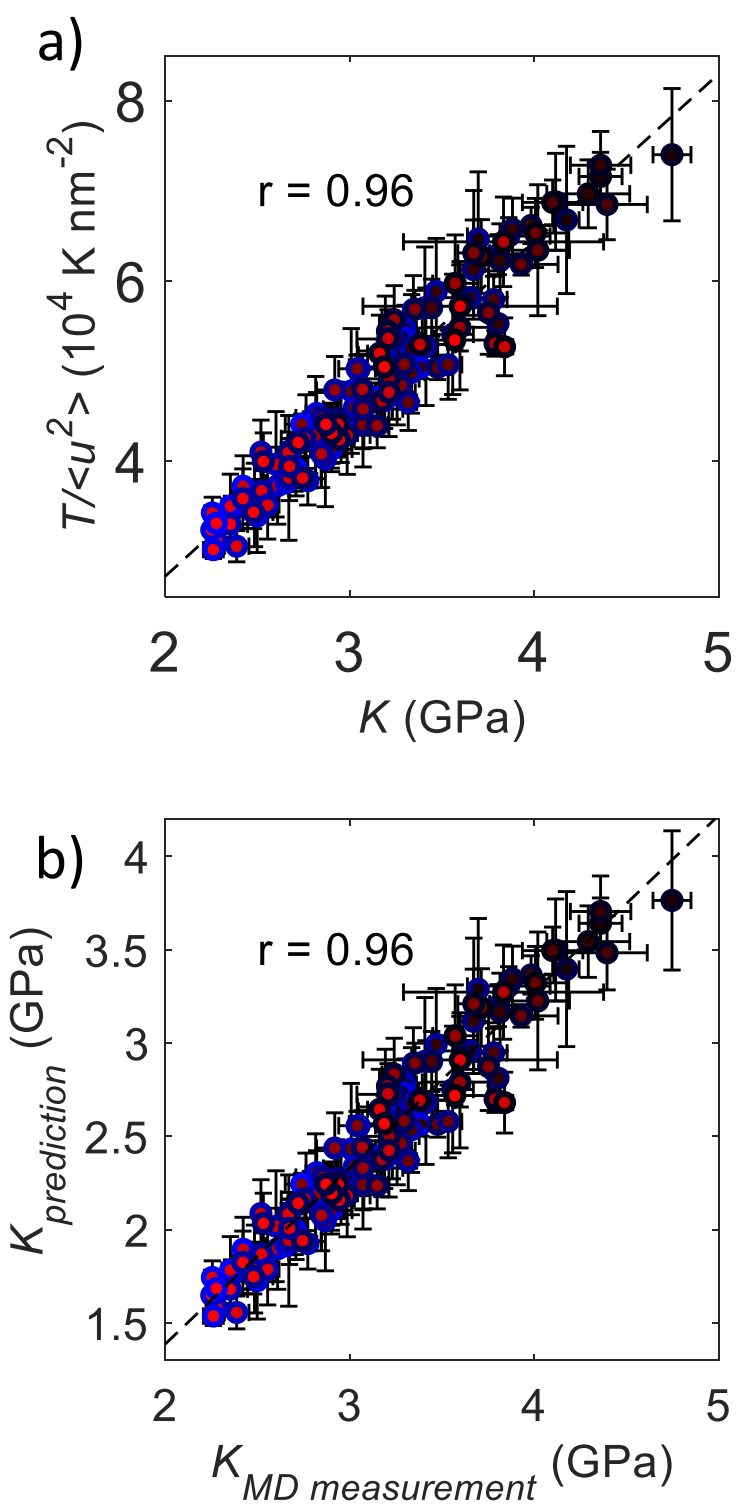

299 Figure 4. a) Correlation plot of local stiffness and the measured bulk moduli. The error bar 300 indicates the standard deviation of the repetition samples. The Pearson correlation coefficient is 301 0.96. b) Comparison of the bulk moduli by MD measurement and predicted through the eq. (8). 302 In both figures, marker face color changes from black to red denoting the rise of temperature, 303 and the marker edge color changes from black to blue denoting the rise of moisture content.

304 The local stiffness can be used to predict the stiffness of material using eq. (8). As shown in 305 Figure $4 \mathrm{~b}$, the prediction agrees well with MD measurement though the absolute values of 
306 prediction are lower than the measurement. The difference between the MD measurement and

307 the prediction may be attributed to the simplifications made in the derivation of eq. (8), such as 308 assuming elasticity, isotropy and homogeneity, the validity of harmonic confinement 309 assumption, the definition of the chain segment size and the accuracy of the method of bulk 310 moduli measurement. To what extent the idealization assumptions affect the predicting power of 311 eq. ( 8 might be the topic of future investigations.

312 As suggested by the form of local stiffness, i.e. $T /\left\langle u^{2}\right\rangle$, moisture content and temperature 313 influence this mechanical property in different ways. Temperature influencing both $T$ and $\left\langle u^{2}\right\rangle$, 314 however, moisture content altering only $\left\langle u^{2}\right\rangle$. Though phenomenologically heat and moisture 315 both induce the macroscopic weakening of material, they impact the molecular mechanics 316 differently.

317 We can also derive a relation between thermal expansion and local stiffness of the material. In 318 Gruneisen theory, ${ }^{45,46}$ based on the existence of an asymmetrical potential energy curve in terms 319 of the distance between atoms, a simple relationship between thermal expansion coefficient and 320 bulk modulus at the macroscale is derived:

$$
\alpha=\frac{\gamma_{G} C_{V}}{V K}
$$

321 where $\alpha, \gamma_{G}, C_{V}, V, K$ are the volumetric thermal expansion coefficient, Gruneisen parameter 322 (defined as the rate of change of vibrational frequency with expansion), specific heat under 323 constant volume, volume and isothermal bulk modulus, respectively. The Gruneisen theory was 324 originally developed for crystals, however, since we see our systems as chain segments moving 325 around their equilibrated location, it is reasonable to extend the theory to our material. The 326 thermal expansion coefficient $\alpha$ is related to thermal strain $\varepsilon_{V}=\alpha \mathrm{d} T$. Combining eq. (8) and eq. 327 (10), we find a relation between volumetric thermal strain $\varepsilon_{V}$ and the local stiffness $T /\left\langle u^{2}\right\rangle$ : 


$$
\varepsilon_{V}=\frac{1-v}{1+v} \frac{\gamma_{G} C_{V} a\left\langle u^{2}\right\rangle}{V k_{B} T}
$$

328 This equation shows that the thermal strain increases with the decrease of local stiffness of the 329 material, which depends on both temperature and moisture content. eq. (11 also shows that 330 several other material properties, including Poisson's ratio, Gruneisen parameter, heat capacity, 331 and temperature, determine the thermal expansion of the material. How these material properties 332 are related to each other and a further deduction of eq. (11 into a simpler form can be a future 333 research topic.

334 3.4 Discussion: Beyond the similarity of heat and moisture

335 We have shown above that both heat and moisture have a similar effect of expanding/swelling 336 and weakening the material. We also showed that the hygric swelling, thermal expansion and 337 mechanical weakening are all related to the increase of the amplitude of segmental motion on the 338 microscale. In this section, we discuss however the differences between the effects of heat and 339 moisture, beyond their similarity.

340 3.4.1 Free straining - the difference between the impacts of moisture and heat

341 We use the data of the free strain $\epsilon_{V}(m, T)$ dependent on temperature $T$ and moisture content $m$

342 in Figure 2a to study the combined effect of heat and moisture. To do so, we introduce three 343 different models, and the results of these models are shown in Table 1, where we used the sum of 344 squared errors (SSE) to evaluate the quality of the model. A first model, called the "first-order 345 independent model" assumes that the free strain is linearly correlated with temperature and 346 moisture content, where independent refers to the fact that the contributions of heat and moisture 347 are additive. In a second model, second-order terms in temperature and moisture content are 348 added, called the "second-order independent model". Finally, in the coupled model a coupling 349 term in temperature and moisture content $m^{*} T$ is added to the first-order independent model. 
350 Comparing the SSE of the different models, we observe that the coupling model provides by far 351 the best results, indicating that the coupling between temperature and moisture content is 352 essential in describing the heat-moisture dependence of free strain. This coupling effect between

353 heat and moisture is a first indication that both factors are not just additive, but that there is an 354 interaction between the two factors.

355 We already have shown that heat facilitates the segmental motions of the polymer chains. 356 Moisture is known to reside as water molecules in between the polymer chains and to interact 357 with the intermolecular interactions between the polymer chains leading to a weakening of the 358 material. As an example of such intermolecular actions, Figure 5 gives the density of the 359 polymer-polymer hydrogen bonds as a function of temperature and moisture content. We 360 observe at the low moisture content $(\mathrm{m}<0.1)$ a sharp decrease in the number of hydrogen bonds 361 indicating the breakage of hydrogen bonds between the chains, which are replaced by water362 polymer hydrogen bonds, leading to a weakening of the material. This weakening of the material 363 by intermolecular interactions of the water molecules in between the polymer chains (breaking of 364 polymer-polymer hydrogen bonds) will, in turn, facilitate lowering the local stiffness and, as 365 such, water molecules in between the chains may be seen as an activator of segmental motions of 366 the chains. This means that the fundamental mechanisms of heat and moisture are different, 367 where moisture has to be seen as an activator, while heat is a direct cause for the segmental 368 motions. This will be further elaborated in the next section. 


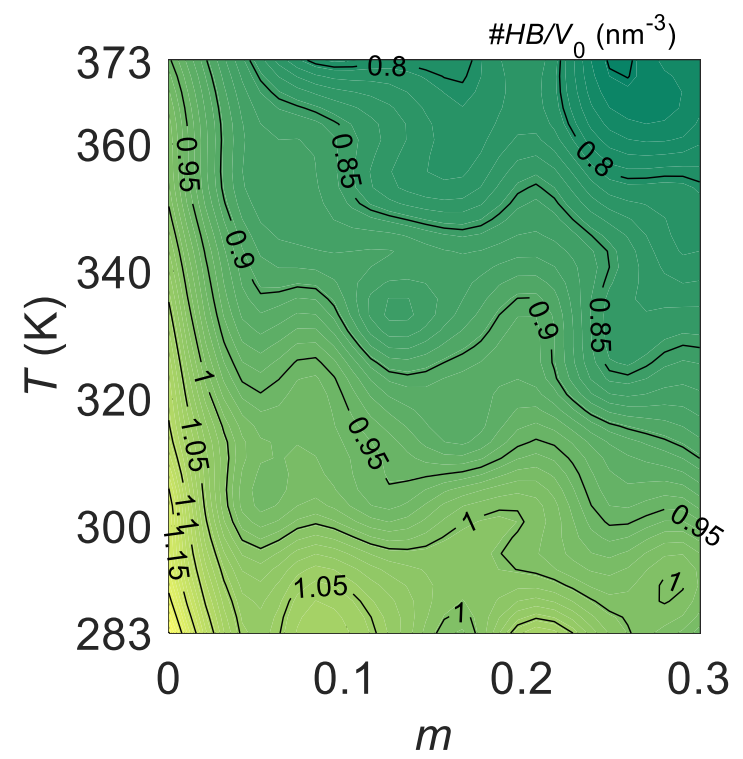

Figure 5. Density of polymer-polymer hydrogen bonds as a function of temperature and 371 moisture content. Units are in $\mathrm{nm}^{-3}$.

\begin{tabular}{lll}
\hline Type of model & Fitting of $\epsilon_{V}(m, T)$ & SSE \\
\hline First order independent model & $1.2 m-7.6 \mathrm{E}^{-4} T-0.24$ & 0.0075 \\
$2^{\text {nd }}$ order independent model & $1.2 m-4.3 \mathrm{E}^{-4} T+0.055 m^{2}+1.8 \mathrm{E}^{-6} T^{2}-0.048$ & 0.0071 \\
coupling model & $0.72 m+3.7 \mathrm{E}^{-4} T+1.4 \mathrm{E}^{-3} m T-0.12$ & 0.0011
\end{tabular}

372 Table 1. Different models for free strain as a function of temperature and moisture content.

373 3.4.2 Primary and secondary bond energy - different impacts of moisture and heat

374 As shown in Figure 2a, uLGN shows an increasing free strain upon increasing temperature,

375 indicating the important role of energetic aspects in the material when being heated. Moreover, 376 as shown in the last sections, the polymer dominates the stiffness of the composite material.

377 Therefore, it is important to analyze the energetic aspects of the uLGN polymer skeleton when 378 the temperature and moisture content increases. The energy terms are obtained through the 379 calculation of potential energy of the polymer skeleton, where the systems consist only of 380 polymer chains after removing all the water molecules from the MD trajectories. 
381 The potential energy of the polymer skeleton consists of two parts: the primary and the 382 secondary bond energy. The primary bond energy includes all the chemical-bond related 383 energies or the so-called "bonded interaction energy" in MD, i.e. bond energy, angle energy, 384 dihedral energy. The secondary bond energy includes all the intermolecular interaction energies 385 or the so-called "non-bonded interaction energy" in MD, i.e. vdW and Coulombic interaction 386 energy. The primary and secondary bond energies of polymer skeleton are shown in Figure 6a 387 and Figure 6b, respectively, where higher and lower values are denoted by yellow and green 388 colors. We note that an increase in potential energy indicates a weakening of the interactions. As 389 shown in Figure 6a, the primary bond energy of polymer skeleton increases mainly with 390 temperature. The contour lines are almost horizontal, indicating an almost negligible influence of 391 moisture content on primary bond energy. 

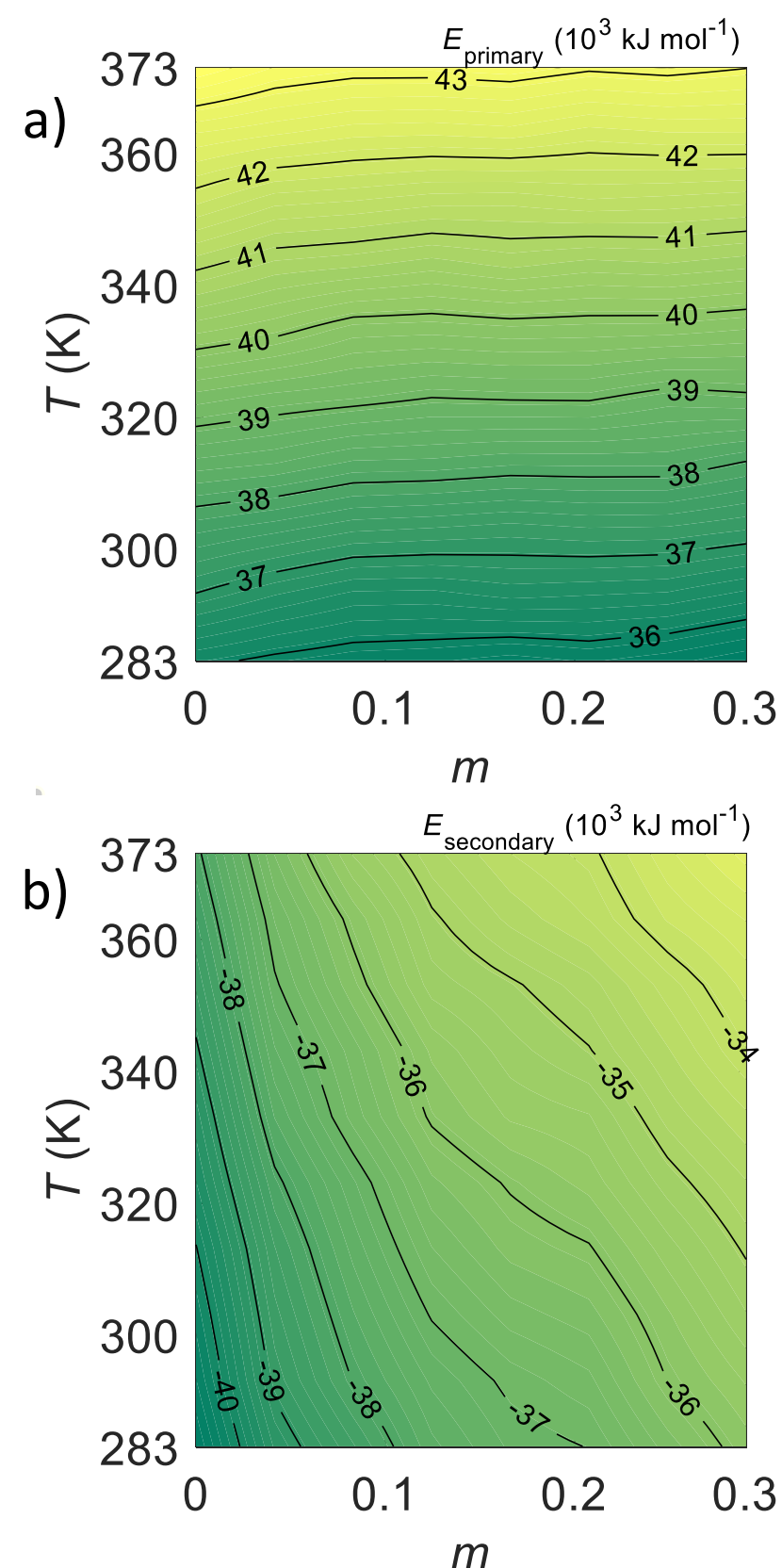

393 Figure 6. Potential energy of polymer skeleton: a) primary bond energy; b) secondary bond 394 energy. The unit of the numbers on the contour lines is $10^{3} \mathrm{~kJ} \mathrm{~mol}^{-1}$.

395 Figure 6b shows the secondary bond energy. We observe that moisture strongly influences the 396 secondary bond energy. The density contour lines of secondary bond energy get looser when 397 moisture content increases, indicating that the influence of moisture is getting weaker as 
398 hydration proceeds. This means that the first adsorbed water molecules have a more important 399 impact on the potential energy, as indicated with the smaller spacing between the counter lines.

400 The temperature also influences the secondary bond energy, but to a less extent than the moisture 401 content, while moisture has only a negligible effect on primary bond energy. This implies that 402 water molecules intervene only with intermolecular interactions, e.g. hydrogen bonds, and can 403 hardly affect chemical bond interactions. This agrees with the former report that water relaxes 404 the charged assemblies by interrupting the attraction between oppositely charged groups of the 405 polymers. ${ }^{47}$

406 Although some similarities between the impact of heat and moisture on free strain and stiffness 407 are observed, in line with the time-temperature-moisture superposition principle proposed by 408 various experimental studies, ${ }^{1-6}$ the effects of heat and moisture have different origins. Heat 409 affects both primary and secondary bonds, while moisture only alters secondary bonds, which is 410 the fundamental difference between heat and moisture effects. Considering the important role of

411 primary and secondary bonds on the stiffness of material, ${ }^{48}$ our findings may have important

412 implications. To mention one example, for thermosetting polymers, where chains are cross-

413 linked into networks and primary bonds play the dominant role, excessive heat will cause the 414 severance of the primary bonds and consequently weakening, ${ }^{49}$ however, the impact of moisture 415 might be limited, which is to be confirmed by future studies.

\section{4. Conclusion}

417 To address the fundamental issue of heat and moisture effects on polymers, this study 418 investigates the prototypical lignin polymer at various temperature and moisture levels using 419 molecular dynamics simulations. In line with previous reports, thermal expansion, hygric 420 swelling and weakening of material occur concomitantly with the increment of temperature and 421 moisture content. The detailed analysis suggests the important role of the coupling between heat 
422 and moisture for the hygric swelling of lignin polymer. To explain the weakening effect, a 423 quantity called local stiffness $T /\left\langle u^{2}\right\rangle$ is introduced. Under the assumptions of harmonic vibration

424 and isotropy, an equation relating microscopic segmental motions $\left\langle u^{2}\right\rangle$ to macroscopic modulus

425 is proposed. This equation allows one to theoretically predict the bulk moduli of material, which

426 are in good agreement with the measurement, supporting that the local stiffness is a good

427 indicator for macroscopic local stiffness. Combined with Gruneisen theory, the thermal 428 expansion is shown to be also related to the local stiffness. The energetic analysis of polymer 429 skeleton shows that, while secondary bonds are influenced by both heat and moisture, the 430 primary bonds are affected by heat alone. This indicates the fundamental difference between

431 moisture and heat, the influences of which have long been assumed to be similar, offering a 432 different view of the so-called time-temperature-moisture superposition principle, based on the 433 molecular level inspection.

434

435 AUTHOR INFORMATION

436 Corresponding Author

437 *E-mail: chi.zhang@empa.ch

\section{$438 \quad$ Funding Sources}

439 The authors acknowledge the support of the Swiss National Science Foundation (SNSF) grant $440 \quad$ No. 162957.

\section{Notes}

442 The authors declare no competing financial interest. 
445 (1) Emri, I.; Pavsek, V. On the Influence of Moisture on the Mechanical Properties of

(3) Fabre, V.; Quandalle, G.; Billon, N.; Cantournet, S. Time-Temperature-Water Content Equivalence on Dynamic Mechanical Response of Polyamide 6,6. Polymer (Guildf). 2018, 137, 22-29. https://doi.org/10.1016/j.polymer.2017.10.067.

(4) Onogi, S.; Sasaguri, K.; Adachi, T.; Ogihara, S. Time-Humidity Superposition in Some Crystalline Polymers. J. Polym. Sci. 1962, 58 (166), 1-17. https://doi.org/10.1002/pol.1962.1205816601.

(5) St. Lawrence, S.; Willett, J. L.; Carriere, C. J. Effect of Moisture on the Tensile Properties of Poly(Hydroxy Ester Ether); 2001; Vol. 42. https://doi.org/10.1016/S00323861(00)00836-3.

(6) Silberstein, M. N.; Pillai, P. V.; Boyce, M. C. Biaxial Elastic-Viscoplastic Behavior of Nafion Membranes. Polymer (Guildf). 2011, $52 \quad$ (2), 529-539. https://doi.org/10.1016/j.polymer.2010.11.032.

(7) Suarez-Martinez, P. C.; Batys, P.; Sammalkorpi, M.; Lutkenhaus, J. L. Time-Temperature and Time-Water Superposition Principles Applied to Poly(Allylamine)/Poly(Acrylic Acid) Complexes. Macromolecules 2019, $52 \quad$ (8), 3066-3074. https://doi.org/10.1021/acs.macromol.8b02512.

(8) Chaléat, C. M.; Michel-Amadry, G.; Halley, P. J.; Truss, R. W. Properties of a Plasticised Starch Blend - Part 2: Influence of Strain Rate, Temperature and Moisture on the Tensile Yield Behaviour. Carbohydr. Polym. 2008, $74 \quad$ (3), 366-371. https://doi.org/10.1016/j.carbpol.2008.03.002.

(9) Williams, M. L.; Landel, R. F.; Ferry, J. D. The Temperature Dependence of Relaxation Mechanisms in Amorphous Polymers and Other Glass-Forming Liquids. J. Am. Chem. Soc. 1955, 77 (14), 3701-3707. https://doi.org/10.1021/ja01619a008.

(10) Harper, B. D.; Rao, J. M.; Kenner, V. H.; Popelar, C. H. Hygrothermal Effects upon Stress Relaxation in a Polyimide Film. J. Electron. Mater. 1997, 26 (7), 798-804. https://doi.org/10.1007/s11664-997-0254-x.

(11) Parodi, E.; Peters, G. W. M.; Govaert, L. E. Prediction of Plasticity-Controlled Failure in Polyamide 6: Influence of Temperature and Relative Humidity. J. Appl. Polym. Sci. 2018, 135 (11), 45942. https://doi.org/10.1002/app.45942.

(12) Valentin, D.; Paray, F.; Guetta, B. The Hygrothermal Behaviour of Glass Fibre Reinforced Pa66 Composites: A Study of the Effect of Water Absorption on Their Mechanical Properties. J. Mater. Sci. 1987, 22 (1), 46-56. https://doi.org/10.1007/BF01160550.

(13) Petridis, L.; Smith, J. C. A Molecular Mechanics Force Field for Lignin. J. Comput. Chem. 2009, 30 (3), 457-467. https://doi.org/10.1002/jcc.21075.

(14) Petridis, L.; Schulz, R.; Smith, J. C. Simulation Analysis of the Temperature Dependence of Lignin Structure and Dynamics. J. Am. Chem. Soc. 2011, 133 (50), 20277-20287. https://doi.org/10.1021/ja206839u.

(15) Vural, D.; Gainaru, C.; O’Neill, H.; Pu, Y.; Smith, M. D.; Parks, J. M.; Pingali, S. V.; 
Mamontov, E.; Davison, B. H.; Sokolov, A. P.; et al. Impact of Hydration and Temperature History on the Structure and Dynamics of Lignin. Green Chem. 2018, 20 (7), 1602-1611. https://doi.org/10.1039/C7GC03796A.

(16) Jin, K.; Qin, Z.; Buehler, M. J. Molecular Deformation Mechanisms of the Wood Cell Wall Material. J. Mech. Behav. Biomed. Mater. 2015, 42, 198-206. https://doi.org/10.1016/j.jmbbm.2014.11.010.

(17) Beste, A. ReaxFF Study of the Oxidation of Lignin Model Compounds for the Most Common Linkages in Softwood in View of Carbon Fiber Production. J. Phys. Chem. A 2014, 118 (5), 803-814. https://doi.org/10.1021/jp410454q.

(18) Xin, D.; Han, Q. Investigation of Moisture Diffusion in Cross-Linked Epoxy Moulding Compound by Molecular Dynamics Simulation. Mol. Simul. 2013, 39 (4), 322-329. https://doi.org/10.1080/08927022.2012.725204.

(19) Vural, D.; Smith, J. C.; Petridis, L. Dynamics of the Lignin Glass Transition. Phys. Chem. Chem. Phys. 2018, 20 (31), 20504-20512. https://doi.org/10.1039/C8CP03144D.

(20) Dorrestijn, E.; Laarhoven, L. J. J.; Arends, I. W. C. E.; Mulder, P. The Occurrence and Reactivity of Phenoxyl Linkages in Lignin and Low Rank Coal. J. Anal. Appl. Pyrolysis 2000, 54 (1-2), 153-192. https://doi.org/10.1016/S0165-2370(99)00082-0.

(21) Pettersen, R. C. The Chemical Composition of Wood; 1984; pp 57-126. https://doi.org/10.1021/ba-1984-0207.ch002.

(22) Abraham, M. J.; Murtola, T.; Schulz, R.; Páll, S.; Smith, J. C.; Hess, B.; Lindah, E. Gromacs: High Performance Molecular Simulations through Multi-Level Parallelism from Laptops to Supercomputers. SoftwareX 2015, 1-2, 19-25. https://doi.org/10.1016/j.softx.2015.06.001.

(23) Oostenbrink, C.; Villa, A.; Mark, A. E.; Van Gunsteren, W. F. A Biomolecular Force Field Based on the Free Enthalpy of Hydration and Solvation: The GROMOS Force-Field Parameter Sets 53A5 and 53A6. J. Comput. Chem. 2004, 25 (13), 1656-1676. https://doi.org/10.1002/jcc.20090.

(24) Malde, A. K.; Zuo, L.; Breeze, M.; Stroet, M.; Poger, D.; Nair, P. C.; Oostenbrink, C.; Mark, A. E. An Automated Force Field Topology Builder (ATB) and Repository: Version 1.0. J. Chem. Theory Comput. 2011, 7 (12), 4026-4037. https://doi.org/10.1021/ct200196m.

(25) Canzar, S.; El-Kebir, M.; Pool, R.; Elbassioni, K.; Malde, A. K.; Mark, A. E.; Geerke, D. P.; Stougie, L.; Klau, G. W. Charge Group Partitioning in Biomolecular Simulation. J. Comput. Biol. 2013, 20 (3), 188-198. https://doi.org/10.1089/cmb.2012.0239.

(26) Koziara, K. B.; Stroet, M.; Malde, A. K.; Mark, A. E. Testing and Validation of the Automated Topology Builder (ATB) Version 2.0: Prediction of Hydration Free Enthalpies. J. Comput. Aided. Mol. Des. 2014, 28 (3), 221-233. https://doi.org/10.1007/s10822-014-9713-7.

(27) Terashima, N.; Kitano, K.; Kojima, M.; Yoshida, M.; Yamamoto, H.; Westermark, U. Nanostructural Assembly of Cellulose, Hemicellulose, and Lignin in the Middle Layer of Secondary Wall of Ginkgo Tracheid. J. Wood Sci. 2009, 55 (6), 409-416. https://doi.org/10.1007/s10086-009-1049-x.

(28) Jorgensen, W. L.; Jenson, C. Temperature Dependence of TIP3P, SPC, and TIP4P Water from NPT Monte Carlo Simulations: Seeking Temperatures of Maximum Density. J. Comput. Chem. 1998, 19 (10), 1179-1186. https://doi.org/10.1002/(SICI)1096987X(19980730)19:10<1179::AID-JCC6>3.0.CO;2-J. 
(29) Yang, H.; Yan, R.; Chen, H.; Zheng, C.; Lee, D. H.; Liang, D. T. In-Depth Investigation of Biomass Pyrolysis Based on Three Major Components: Hemicellulose, Cellulose and Lignin. Energy \& Fuels 2006, 20 (1), 388-393. https://doi.org/10.1021/ef0580117.

(30) Jackson, C. L.; McKenna, G. B. The Melting Behavior of Organic Materials Confined in Porous Solids. J. Chem. Phys. 1990, 93 (12), 9002-9011. https://doi.org/10.1063/1.459240.

(31) Roduner, E. Thermodynamics of Finite Size Systems. In Nanoscopic Materials; Royal Society of Chemistry: Cambridge; pp 119-162. https://doi.org/10.1039/978184755763600119.

(32) Kulasinski, K.; Keten, S.; Churakov, S. V.; Guyer, R.; Carmeliet, J.; Derome, D. Molecular Mechanism of Moisture-Induced Transition in Amorphous Cellulose. ACS Macro Lett. 2014, 3 (10), 1037-1040. https://doi.org/10.1021/mz500528m.

(33) Luzar, A.; Chandler, D. Structure and Hydrogen Bond Dynamics of Water-Dimethyl Sulfoxide Mixtures by Computer Simulations. J. Chem. Phys. 1993, 98 (10), 8160-8173. https://doi.org/10.1063/1.464521.

(34) Soper, A. K.; Phillips, M. G. A New Determination of the Structure of Water at $25^{\circ} \mathrm{C}$. Chem. Phys. 1986, 107 (1), 47-60. https://doi.org/10.1016/0301-0104(86)85058-3.

(35) Teixeira, J.; Bellissent-Funel, M. C. Dynamics of Water Studied by Neutron Scattering. $J$. Phys. Condens. Matter 1990, 2. https://doi.org/10.1088/0953-8984/2/S/011.

(36) Riggleman, R. A.; Douglas, J. F.; de Pablo, J. J. Antiplasticization and the Elastic Properties of Glass-Forming Polymer Liquids. Soft Matter 2010, 6 (2), 292-304. https://doi.org/10.1039/B915592A.

(37) van Zanten, J. H.; Rufener, K. P. Brownian Motion in a Single Relaxation Time Maxwell Fluid. Phys. Rev. E 2000, 62 (4), 5389-5396. https://doi.org/10.1103/PhysRevE.62.5389.

(38) Debye, P. Interferenz von Röntgenstrahlen Und Wärmebewegung. Ann. Phys. 1913, 348 (1), 49-92. https://doi.org/10.1002/andp.19133480105.

(39) Waller, I. Zur Frage Der Einwirkung Der Wärmebewegung Auf Die Interferenz von Röntgenstrahlen. Zeitschrift für Phys. 1923, 17 (1), 398-408. https://doi.org/10.1007/BF01328696.

(40) Price, D. L.; Fernandez-Alonso, F. An Introduction to Neutron Scattering; 2013; pp 1136. https://doi.org/10.1016/B978-0-12-398374-9.00001-2.

(41) Xia, W.; Song, J.; Jeong, C.; Hsu, D. D.; Phelan, F. R.; Douglas, J. F.; Keten, S. EnergyRenormalization for Achieving Temperature Transferable Coarse-Graining of Polymer Dynamics. $\quad$ Macromolecules $\quad \mathbf{2 0 1 7}, \quad 50 \quad$ (21), 8787-8796. https://doi.org/10.1021/acs.macromol.7b01717.

(42) Grimvall, G. Thermophysical Properties of Materials; Elsevier, 1999. https://doi.org/10.1016/B978-0-444-82794-4.X5000-1.

(43) Zaccai, G. How Soft Is a Protein? A Protein Dynamics Force Constant Measured by Neutron Scattering. Science (80-. $) . \quad 2000,288$ (5471), 1604-1607. https://doi.org/10.1126/science.288.5471.1604.

(44) Salmén, L. Micromechanical Understanding of the Cell-Wall Structure. Comptes Rendus Biol. 2004, 327 (9-10), 873-880. https://doi.org/10.1016/j.crvi.2004.03.010.

(45) Tabor, D. Gases, Liquids and Solids: And Other States of Matter; Cambridge University Press, 1991.

(46) Grüneisen, E. Theorie Des Festen Zustandes Einatomiger Elemente. Ann. Phys. 1912, 344 (12), 257-306. https://doi.org/10.1002/andp.19123441202. 
(47) Zhang, Y.; Batys, P.; O’Neal, J. T.; Li, F.; Sammalkorpi, M.; Lutkenhaus, J. L. Molecular Origin of the Glass Transition in Polyelectrolyte Assemblies. ACS Cent. Sci. 2018, 4 (5), 638-644. https://doi.org/10.1021/acscentsci.8b00137.

(48) Soboyejo, W. Mechanical Properties of Engineered Materials; 2002. https://doi.org/10.1201/9780203910399.

(49) Callister Jr, W. D. Materials Science and Engineering - An Introduction (5th Ed.); 2000; Vol. 47. https://doi.org/10.1108/acmm.2000.12847aae.001. 
589 Disentangling Heat and Moisture Effects on

590 Biopolymer Mechanics

591 Chi Zhang ${ }^{\ddagger *}$, Ali Shomali ${ }^{\dagger}$, Robert Guyer ${ }^{*}$, Sinan Keten ${ }^{\S}$, Benoit Coasne ${ }^{\Perp}$, Dominique Derome ${ }^{\ddagger}$,

592 Jan Carmeliet

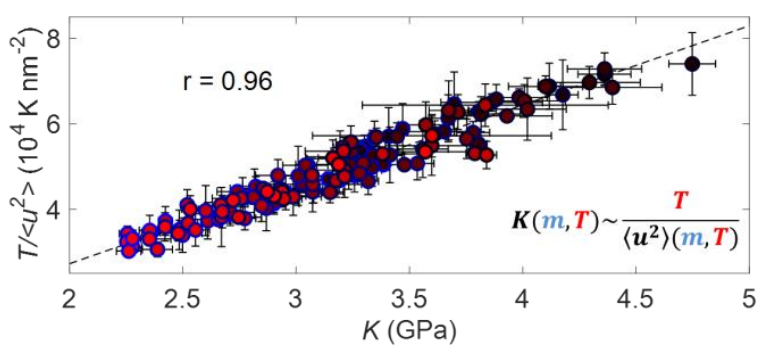

594

31 